\title{
Análise da mobilização articular da cervical em indivíduos com cefaleia do tipo tensão
}

Analysis of active and passive joint repositioning in individuals with tension-type headache

FisiSenectus. Unochapecó Ano 6, n. 2 - Jul/Dez. 2018 p. $16-25$

Ana Paula Barcellos Karolczak. anapbk@gmail.com

Fisioterapeuta. Doutora em Saúde Coletiva. Docente do Curso de Fisioterapia da Universidade do Vale do Rio dos Sinos (UNISINOS).

Tissiani Morimoro. tissi.morimoto@gmail.com

Fisioterapeuta. Mestre em Saúde Coletiva. Docente do Curso de Fisioterapia do Centro Universitário Ritter dos Reis (UniRitter).

Rodrigo Daniel do Nascimento. rodrigo.donascimento@hotmail.com

Fisioterapeuta. Especialista em Fisioterapia Ortopédica e Traumatológica.

\section{Resumo}

Introdução: A cefaleia é uma das doenças do sistema nervoso mais comuns, podendo acarretar incapacidades. Seu tratamento pode envolver terapias não medicamentosas, dentre elas a fisioterapia. Objetivo: avaliar os efeitos agudos das técnicas de SNAGS C1/C2 e auto SNAGS C1/C2, em indivíduos com cefaleia tipo tensão (CTT). Metodologia: Estudo experimental, com 15 voluntários universitários divididos em dois grupos: Mobilização Passiva (GP, $n=8)$ e Mobilização Ativa ( $G A, n=7)$. A coleta foi realizada em única intervenção individual durante 30 minutos, sendo avaliação (avaliação da dor com a Escala Visual Analógica da Dor; avaliação da amplitude de movimento (ADM) de flexão, extensão, flexão lateral à direita e esquerda, e rotação à direita e esquerda da cervical), aplicação da técnica (três séries de 20 repetições, ativa ou passiva) e reavaliação. A coleta foi iniciada após a aprovação do Comitê de Ética em Pesquisa (16/079). Resultados: Verificou-se que as comparações entre grupos não apontaram diferenças significativas para dor (GP: 5,4 1,$3 ; G A: 4,4 \pm 1,3 ; p=0,211$ antes; GP: $3,8 \pm 1,4 ; G A: 2,7 \pm 1,7$ após; $p=0,253)$. Os resultados referentes à $A D M$ apontaram que a flexão cervical aumentou significativamente nos dois grupos, GP $(47,5 \pm 10,8 ; 50,9 \pm 11,2 ; p=0,027)$ e $G A(47,9 \pm 8,4 ; 53,6 \pm 10,5 ; p=0,025)$. No que se refere à rotação cervical, houve aumento tanto na comparação entre grupos, quanto intragrupo. Rotação à direita, GP $(36,3 \pm 5,9 ; 41,3 \pm 4,9 ; p=0,011)$ e $G A(39,9 \pm 3,5 ; 46,0 \pm 3,1 ; p=0,027)$. Rotação à esquerda, $\operatorname{GP}(37,8 \pm 6,2 ; 42,4 \pm 7,5 ; p=0,011)$, e GA $(43,9 \pm 3,8 ; 48,6 \pm 4,8 ; p=0,036)$. Conclusão: Após a intervenção houve redução na dor e aumento da amplitude de movimento cervical em ambas as técnincas.

\section{Palavras-chave}

Fisioterapia; Terapia Manual; Cefaleia; Articulação Atlantoaxial.

\section{Abstract}

Introduction: Headache is one of the most common diseases of the nervous system, which can lead to incapacities. The treatments for headache involve drug and non-drug therapies, among them physiotherapy. 
Objective: to evaluate the immediate effects of the SNAGS C1/C2 and self SNAGS C1/C2 techniques in individuals with tension-type headache (TTH). Methodology: Experimental study with 15 groups of university volunteers in two groups: Passive Mobilization ( $P G, n=8$ ) and Active Mobilization ( $A G, n=7)$. The collection was performed in a single individual intervention with a duration of 30 minutes was performed, being evaluation (pain evaluation with the Visual Analog Pain Scale, flexion extension, flexion extension, right and left lateral flexion, and right rotation and cervical left), technique application (three sets of 20 repetitions, active or passive) and reevaluation. The collection was initiated after the approval of the Ethics Committee in Research (16/079). Results: the sample consisted of 15 individuals (Passive-PG, $n=8$ and Active-AG, $n=7$ ). Comparisons between groups did not show significant differences for pain, indicating that before (PG: $5.4 \pm 1.3$, AG: $4.4 \pm 1.3, p=0.211$ ), and after the intervention (PG: $3.8 \pm 1.4, A G: 2.7 \pm 1.7, p=0.253$ ), there was a decrease. Results for ROM showed that cervical flexion increased significantly in both groups, PG $(47.5 \pm 10.8,50.9 \pm 11.2$, $p=0.027)$ and $A G(47.9 \pm 8.4,53.6 \pm 10.5, p=0.025)$. With regard to cervical rotation, there was an increase both in the comparison between groups and in the intragroup. Rotation at right, $P G(36.3 \pm 5.9,41.3 \pm 4.9, p=0.011)$ and $A G(39.9 \pm 3.5,46.0 \pm 3.1, p=0.027)$. Rotation at left, PG $(37.8 \pm 6.2,42.4 \pm 7.5, p=0.011)$, and AG (43.9 \pm 3.8 , $48.6 \pm 4.8, p=0.036)$. Conclusion: After the application of the SNAGS C1/C2 and self SNAGS C1/C2 techniques, there was a reduction in pain and an increase in the range of cervical movement.

\section{Keywords}

Physiotherapy; Manual therapy; Headache; Atlantoaxial Joint.

\section{Introdução}

$\infty \times \infty \times \infty \times \infty \times \infty \times \infty \times \infty \times \infty \times \infty \times \infty \times \infty \times \infty \times \infty)$

A cefaleia é uma das doenças do sistema nervoso mais comuns, podendo acarretar incapacidades, caracterizando-se como um problema de saúde pública. Sua prevalência é de aproximadamente $51 \%$ entre adultos e $5 \%$ em adolescentes. 0 subtipo mais frequente é a cefaleia do tipo tensão (CTT), que se caracteriza como uma dor bilateral, constante, com caráter constritivo, de intensidade leve a moderada ${ }^{1-3}$. A epidemiologia dos distúrbios da dor de cabeça é documentada parcialmente no mundo. Muitos estudos investigam a enxaqueca, entretanto, os dados sobre a CTT, o subtipo mais frequente, são relativamente escassos ${ }^{2}$.

Para seu diagnóstico, é importante considerar a frequência em que a CTT ocorre. Entre os critérios, estão a duração de pelo menos 30 minutos por dia, a ocorrência em 15 dias por mês, por mais de três meses. A CTT é detectada em todas as faixas etárias, sendo mais frequente em pessoas de meia idade ${ }^{1}$.

A dor originada pela CTT está localizada nos músculos da região frontal, temporal ou frontotemporal, frequentemente com um componente occipital. Vários são os fatores relacionados com o surgimento da CTT. Disfunções posturais combinadas com fatores emocionais são apontados como principais desencadeantes do quadro álgi$\mathrm{CO}^{4}$. A fisiopatologia da CTT é descrita por alguns autores como uma sobrecarga muscular, provocada por contração isométrica sustentada com consequente redução do aporte sanguíneo e isquemia. A sobrecarga muscular favorece a liberação de neurotrasmissores que agem provocando maior recrutamento de fibras musculares e, consequentemente, aumentam a tensão e retração muscular, gerando dor irradiada e alterações nos movimentos cervicais ${ }^{4}$.

Os tratamentos para a CTT envolvem terapias medicamentosas e não medicamentosas. Dentre as terapias não medicamentosas, destaca-se a fisioterapia, que pode se configurar como uma importante abordagem para minimizar este problema, atuando no controle sintomatológico ${ }^{5,6}$. Uma das técnicas para o controle álgico é a terapia manual, e dentro deste contexto, Mulligan, em 1987, propôs a técnica "sustained natural apophyseal glide (SNAGS)", em português "deslizamento natural apofisário sustentado", para as situações onde há restrição de movimento da coluna cervical por dor. Esta técnica proporciona o reposicionamento articular no nível cervical nos casos de redução da amplitude de movimento (ADM) na rotação 
das vértebras $\mathrm{C} 1 / \mathrm{C2}$, a qual está frequentemente relacionada com a $\mathrm{CTT}^{7}$.

O objetivo deste estudo, portanto, foi avaliar os efeitos agudos das mobilizações de Mulligan", de forma passiva (SNAGS C1/C2) e ativa (auto SNAGS C1/C2) em indivíduos com CTT.

\section{Metodologia}

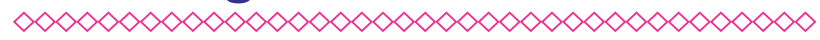

Este estudo caracteriza-se como quantitativo de caráter quase experimental. A população foi composta por estudantes universitários que apresentaram sinais e sintomas de CTT e concordaram em participar e assinaram o Termo de Consentimento Livre e Esclarecido (TCLE). Foram incluídos na pesquisa indivíduos de ambos os sexos, com idades entre 18 e 40 anos, com dez ou mais crises de CTT ao ano e que apresentaram redução na ADM de rotação cervical maior ou igual a 10 graus, sendo considerado parâmetro de normalidade 55 graus $^{8}$. Foram excluídos indivíduos que apresentaram qualquer outro tipo de cefaleia que não se enquadrava nos critérios de diagnóstico da CTT; mulheres grávidas ou no período menstrual; indivíduos que sofreram algum trauma ou doença na região cervical, como fraturas, tumores, infecções, entre outras; indivíduos em que o teste da artéria vertebrobasilar foi positivo; e indivíduos que se medicaram para o controle da dor.

$\mathrm{Na}$ Classificação Internacional das Cefaleias, os critérios de diagnósticos são descritos para cada subtipo e subforma. 0 paciente com CTT deve apresentar ao menos dez episódios no período de um ano com duração de 30 minutos a 15 dias a cada crise, apresentando no mínimo duas das quatro características e sintomas a seguir: A - dor tipicamente bilateral; $B$ - com caráter de pressão ou aperto (não pulsátil); $C$ - de intensidade leve a moderada; e D - não agravada com a atividade física de rotina. 0 paciente não deve apresentar náuseas e/ou vômitos e apenas um dos seguintes sintomas pode estar presente: fotofobia ou fonofobia. É importante destacar que este tipo de cefaleia é classificada como disfuncional, não podendo ser considerada como dores somáticas, neuropáticas ou viscerais ${ }^{9}$.
A seleção da amostra foi feita por conveniência e a divulgação por meio de cartazes afixados em murais e folhetos distribuídos aos estudantes universitários, cuja função era convidar para participarem da pesquisa aqueles que apresentavam queixas de dores de cabeça. Em um processo de três etapas, foi feita a triagem dos indivíduos que se prontificaram a participar. Na primeira etapa, o contato foi por e-mail ou telefone, na segunda ocorreu a marcação de horário no dia da crise álgica e na terceira etapa ocorreu a avaliação presencial. Os voluntários foram encaminhados à Clínica Escola de Fisioterapia da Universidade do Vale do Rio dos Sinos, no dia que apresentaram crise álgica. Inicialmente, o pesquisador preencheu e aplicou o questionário de avaliação para seleção da amostra e verificou se o voluntário enquadrava-se nos critérios de diagnóstico da CTT. Este questionário continha itens como: localização, intensidade e características da dor; periodicidade; descrição postural de rotina na aula ou trabalho; fatores agravantes; e se fazia uso de medicamentos para alívio da dor ${ }^{10,11}$. Após o preenchimento do questionário, foi aplicado o teste da artéria vertebrobasilar como forma de investigação quanto aos sinais e sintomas de que tipo de cefaleia que cada voluntário se enquadrava ${ }^{12}$.

Para a avaliação subjetiva da dor, utilizou-se a Escala Visual Analógica da Dor (EVA). A EVA é um instrumento de avaliação subjetiva da dor em que o paciente, em uma linha reta graduada de 0 a 10 $\mathrm{cm}$, relata a sensação, cuja extremidade inicial (zero) corresponde à ausência da dor e a extremidade final (dez) à dor máxima ${ }^{6}$.

0 instrumento Goniômetro Universal (Carci®) foi utilizado para medir a ADM articular da região cervical: flexão, extensão, flexão lateral à direita e esquerda, e rotação à direita e esquerda. Os valores normais de ADM considerados foram: flexão - 0 a 65 graus, extensão - 0 a 50 graus, flexão lateral bilateral - 0 a 40 graus e rotação bilateral - 0 a 55 graus $^{8}$.

Tanto a EVA quanto a ADM foram verificadas antes e após a intervenção fisioterapêutica. Os voluntários foram divididos aleatoriamente em dois grupos: Grupo Passiva (GP) - aplicação da técnica passiva - SNAGS C1/C2; e Grupo Ativa (GA) - orientado quanto à aplicação da técnica ativa - AUTO SNAGS C1/C2. 
A coleta de dados foi realizada em uma única intervenção individual com duração de 30 minutos, incluindo avaliação, aplicação da técnica (três séries de 20 repetições, ativa ou passiva) e reavaliação. A coleta foi iniciada somente após a aprovação do Comitê de Ética em Pesquisa da Unisinos ( $N^{0}$ 16/079), estando de acordo com os preceitos da Resolução 466/12 do Conselho Nacional de Saúde.

As análises foram realizadas por meio de estatística descritiva (distribuição absoluta e relativa), bem como por média, desvio padrão e mediana. 0 estudo da distribuição de dados das variáveis contínuas ocorreu pelo teste de Shappiro Wilk. Para a análise bivariada entre variáveis categóricas, foi utilizado o teste Exato de Fisher $\left(c^{2}\right)$. Nas comparações onde se detectou algum grau de dependência, foi aplicado o teste de Cohran (múltiplas escolhas). Para a comparação das variáveis contínuas, foram aplicados os testes t-Student e Mann Whitney $U$.

A análise estatística dos dados foi feita por meio de avaliação inferencial para amostras independentes utilizando o software Statistical Package for Social Sciences (SPSS) versão 22.0, sen-

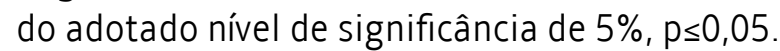

\section{Resultados}

$\infty<\infty<\infty<\infty<\infty<\infty<\infty<\infty<\infty<\infty<\infty<\infty<\infty<\infty<\infty<\infty$

\section{Caracterização da Amostra e Critérios de Diagnóstico da CTT}

A amostra foi composta inicialmente por 18 voluntários. Dois voluntários foram excluídos por apresentarem outro tipo de cefaleia, com característica latejante e pulsátil; e um terceiro por não apresentar redução mínima de 10 graus de ADM na rotação cervical. Dessa forma, a amostra estudada foi composta por 15 indivíduos, pertencentes a dois grupos independentes, referente à técnica aplicada: técnica SNAGS C1/C2 - Passiva (GP), $53,3 \%(n=8)$ e técnica Auto SNAGS C1/C2 - Ativa (GA), 46,7\% ( $n=7)$.

Na caracterização do perfil da amostra, predominou o sexo feminino com média de idade de $26,4 \pm 7,5$ anos no GA e de $25,7 \pm 5,1$ anos GP (Tabela 1 ).
Quanto aos critérios de diagnósticos da CTT, os resultados mostraram que a postura habitual diária mais relatada foi a sentada, tanto no GP, $75,0 \%$ $(n=6)$ quanto no $G A, 57,1 \%(n=4)$. 0 número de crises, no período de um ano, mostrou-se significativamente mais elevado $(p=0,017)$ no $G A(72,8 \pm 29,8$ crises/ano) quando comparado ao GP $(31,5 \pm 26,7$ crises/ano). Em relação à duração das crises, não houve diferença significativa ( $p>0,999)$ (Tabela 2).

A comparação das regiões acometidas não mostrou diferença significativa entre os grupos $(p=0,156)$, predominando a região frontal relatada por todos os investigados do GP, 100,0\% (n=8), e por $85,7 \%(n=6)$ dos investigados do GA (Tabela 2$)$.

Em relação ao tipo de dor, não houve diferença significativa entre os grupos $(p=0,103)$, sendo as dores do tipo pressão (GP - 75\% - n=6; GA - 57,1\% $-n=4)$ e aperto (GP - 37,5\% - $n=3 ; G A-57,1 \%-n=4)$ as mais relatadas (Tabela 2 ).

\section{Avaliação da Dor}

Os resultados referentes à avaliação da dor, antes e após a aplicação das técnicas, mostraram que não houve diferença significativa entre os grupos, indicando que, tanto na EVA antes da intervenção (GP: $5,4 \pm 1,3 ; G A: 4,4 \pm 1,3 ; p=0,211$ ) quanto após

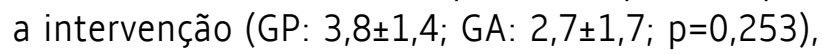
o grau de dor era semelhante dois grupos (Teste t-Student para grupos independentes assumindo homogeneidade de variâncias). No entanto, na comparação intragrupo, observou-se diferença estatisticamente significativa na redução da dor antes e após intervenções (GP redução de 29,6\%, $p=0,010 ; G A$ redução de 38,6\%; $p=0,016$ ) (Teste t-Student para amostras pareadas).

\section{Avaliação da Amplitude de Movimento}

Na ADM de flexão cervical encontrou-se diferença significativa nas comparações intragrupo, tanto no GP (A: $47,5 \pm 10,8$ graus; R: $50,9 \pm 11,2$ graus; $p=0,027)$ quanto no $G A(A: 47,9 \pm 8,4$ graus; R: $53,6 \pm 10,5$ graus; $p=0,025$ ) (Tabela 3 ).

Em relação à flexão lateral $D$, na comparação intragrupo, ocorreu aumento significativo da média de ADM no GP (A: $34,1 \pm 5,2$ graus; $R$ : $37,8 \pm 2,7$ graus; $p=0,043$ ) (Tabela 3 ).

Na comparação intragrupo, a ADM de rotação à $D$ aumentou significativamente, tanto no GP 
(A: $36,3 \pm 5,9$ graus; $R: 41,3 \pm 4,9$ graus; $p=0,011$ ) quanto no $G A$ (A: $39,9 \pm 3,5$ graus; $R$ : $46,0 \pm 3,1$ graus; $p=0,027)$. Em relação a comparação entre grupos, a diferença significativa ocorreu na reavaliação, pois a média no GA se mostrou mais elevada $(p=0,047)$. Quanto à rotação e as comparações intragrupo mostraram-se significativas, tanto no GP (A: $37,8 \pm 6,2$ graus; $R: 42,4 \pm 7,5$ graus; $p=0,011$ ) quanto no $G A(A: 43,9 \pm 3,8$ graus; $R: 48,6 \pm 4,8$ graus; $\mathrm{p}=0,036)$ (Tabela 3).

\section{Discussão}

$\infty \times \infty \times \infty \times \infty \times \infty \times \infty \times \infty \times \infty \times \infty \times \infty \times \infty \times \infty \times \infty)$

De forma semelhante ao presente estudo, a literatura indicou que as mulheres são mais acometidas do que os homens. Em relação à idade, Fumal e Schoenen ${ }^{13}$ encontraram que a prevalência de CTT é maior na faixa etária dos 40-4913, idade esta superior a do presente estudo. Deve-se destacar que a amostra do presente estudo foi composta por estudantes universitários, caracterizando uma faixa etária menor. Já Mendes et al. ${ }^{14}$, em estudo sobre o uso da terapia manual e do alongamento em indivíduos com CTT, encontraram idade média de 27,3 anos de idade, corroborando com o presente estudo ${ }^{15}$.

Em relação aos critérios de diagnóstico da CTT, a sensação de dor com característica bilateral mostrou-se presente, e o tipo de dor caracterizada como pressão e aperto. Estes achados estão de acordo com os critérios de diagnóstico das CTT presentes na literatura ${ }^{9,13}$.

No que se refere à intensidade da dor, houve redução significativa intragrupo após aplicação das técnicas. A técnica passiva SNAGS C1/C2 é altamente eficaz no alívio da dor em uma única aplicação. Essa diminuição da intensidade da dor é explicada pela teoria da falha reposicional, que ocasiona uma restrição de movimentos da coluna cervical, gerando a dor. Dessa forma, a diminuição da dor se dá pela restauração do alinhamento articular normal $1^{7,16}$.

Em relação à técnica ativa, o estudo feito por Hall et al. ${ }^{17}$ demonstrou a eficácia do Auto SNAGS C1/C2 na redução da dor de cabeça cervicogênica. Participaram 32 indivíduos com dor de cabeça e limitação na ADM cervical em flexão e rotação, divididos em dois grupos: técnica Auto SNAGS C1/ C2; e placebo. Os indivíduos do grupo intervenção foram orientados a aplicar a técnica ativa, diariamente, duas vezes ao dia. Após as orientações e a prática inicial, houve redução significativa da intensidade da dor entre os grupos $(p<0,001)$, ao longo de um período de um ano.

Nos resultados para ADM cervical, os movimentos de flexão e rotação apresentaram aumento significativo da ADM intragrupo, em ambos os grupos avaliados. Já na comparação entre grupos, o GA apresentou maior ganho de ADM. Esses resultados corroboram com estudo no qual foi verificado aumento de 15 graus de ADM no grupo Auto SNAGS C1/C2, e cinco graus no grupo placebo $(p<0,001)^{17}$.

A biomecânica da articulação atlantoaxial (C1/ (2) é a principal responsável pelo movimento de rotação cervical. Quando avaliada uma redução na ADM de rotação cervical, o paciente apresenta uma restrição de movimento por falha reposicional articular. Com isso, as técnicas utilizadas podem explicar o ganho de ADM de rotação cervical encontrado no presente estudo, uma vez que favorecem o reposicionamento articular buscando a melhora funcional e a diminuição da dor, sendo considerada, atualmente, uma das técnicas mais eficazes no tratamento de disfunções musculoesqueléticas ${ }^{7}$.

Nesta pesquisa, foi utilizado o método de goniometria para avaliar a ADM cervical. Na literatura, diversos estudos utilizaram o teste de flexão e rotação cervical (TFR) para mensurar limitações de movimentos. Embora este estudo não tenha como objetivo comparar os métodos de avaliação da ADM, os achados clínicos de ambos os métodos apontam para redução da ADM cervical em indivíduos com cefaleia ${ }^{17-20}$.

Hall et al., em um estudo com o objetivo de comparar os resultados do TFR entre indivíduos com provável cefaleia cervicogênica $(n=20)$, enxaqueca sem aura $(n=20)$ e múltiplas formas (MF) de dor de cabeça $(n=20)$, verificaram que a rotação foi significativamente menor no grupo de cefaleia cervicogênica quando comparada com os grupos da enxaqueca $(p<0,001)$ e MF $(p \leq 0,001)^{20}$.

Autilização da goniometria para mensuração da ADM pode apresentar algumas diferenças quando 
comparada com o TFR. No entanto, a goniometria é considerada de alta validade para a mensuração de ADM em disfunções musculoesqueléticas, principalmente da coluna cervical, servindo como base para avaliação da eficácia de intervenções fisioterapêuticas ${ }^{21,22}$.

No presente estudo, a amostra foi selecionada por conveniência, o que pode se configurar como limitação, além da ausência de follow up dos voluntários para se analisar os efeitos posteriores.

\section{Conclusão}

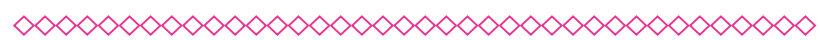

Após a aplicação das técnicas de reposicionamento articular, SNAGS C1/C2 e Auto SNAGS C1/ $\mathrm{C} 2$, em indivíduos com CTT, houve como efeito imediato a redução da dor e o aumento da ADM cervical, principalmente de rotação, o que favoreceu o reposicionamento articular (C1/C2) e a funcionalidade de pacientes com crises de CTT.

\section{Referências}

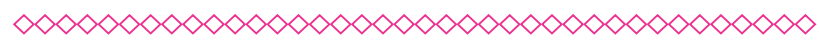

1. Pihut M, Ferendiuk E, Szewczyk M, Kasprzyk $K$, Wieckiewicz $M$. The efficiency of botulinum toxin type A for the treatment of masseter muscle pain in patients with temporomandibular joint dysfunction and tension-type headache. J Headache Pain. 2016;17(29):1-6. doi: 10.1186/ s10194-016-0621-1.

2. Stovner LJ, Hagen K, Jensen R, Katsarava Z, Lipton RB, Scher AI, et al. The global burden of headache: a documentation of headache prevalence and disability worldwide. Cephalalgia. 2007;27:193-210. doi:10.1111/j.14682982.2007.01288.x.

3. Speciali JG, Dach F. Temporomandibular dysfunction and headache disorder. Headache. 2015;55(S1):72-83. doi: 10.1111/head.12515.

4. Medeiros AA De, Lima BRD de A, Siqueira DF de. A eficácia da fisioterapia manual na cefaléia tensional: uma revisão sistemática. Ter Man. 2012;10(47):100-4. doi: 10.9736/TerMan.v10. n47.73.
5. Rabello GD, Forte LV, Galvão ACR. Avaliação clínica da eficácia da combinação paracetamol e cafeína no tratamento da cefaléia tipo tensão. Arq Neuropsiquiatr. 2000;58(1):90-8. doi: 10.1590/ S0004-282X2000000100014.

6. Almeida RS, Gomes V, Gaullier CDM, Dames KK, Nogueira LAC. Efeitos da terapia manual na cefaleia do tipo cervicogênica: uma proposta terapêutica. Acta Fisiatr. 2014;21(2):53-7. doi: 10.5935/0104-7795.20140012.

7. Mulligan BR. Terapia manual: Técnicas NAGS SNAGS - MWM e suas variantes. 5th ed. São Paulo: Premier; 2009.

8. Marques AP. Manual de goniometria. 2nd ed. São Paulo: Manole; 2003.

9. Society IH (IHS). Classificação internacional das cefaleias. Tradução de Sociedade Portuguesa de Cefaleias. 3rd ed. Portugal: ICHD; 2013.

10. Halal IS, Fernandes JG. Cefaléia. In: Duncan, BB et al. Medicina ambulatorial: condutas clínicas em atenção primária. 2nd ed. Porto Alegre: Artmed; 1996. p. 708-12.

11. Galvão ACR. Cefaléias primárias. In: Teixeira, MJ; Figueiró, JAB. Dor: epidemiologia, fisiopatologia, avaliação, síndromes dolorosas e tratamento. São Paulo: Moreira Junior; 2001. p. 283-93.

12. Cipriano JJ, Vissoky J. Manual fotográfico de testes neurológicos e ortopédicos. 5th ed. São Paulo: Artmed; 2012.

13. Fumal A, Schoenen J. Tension-type headache: current research and clinical management. Lancet Neurol. 2008;7:70-83. doi: 10.1016/S14744422(07)70325-3.

14. Loder E, Rizzoli P. Tension-type headache. BMJ. 2008;336:88-92. doi: 10.1136/ bmj.39412.705868.AD.

15. Mendes MRP, Silva AN, Amaral JT. Uso da terapia manual e do alongamento em indivíduos com cefaleia tensional. Linkania. 2014;1(8):102-25.

16. Alves CM, Junior AJC, Vieira TC, Cruz RS da. Efetividade do conceito Mulligan na dor cervical e lombar: estudo com intervanção. 
Estudos. 2013;40(2):177-86. doi: 10.18224/est. v40i2.2720.

17. Hall T, Chan HT, Christensen L, Odenthal B, Wells C, Robinson K. Efficacy of a C1-C2 Selfsustained Natural Apophyseal Glide (SNAG) in the Management of Cervicogenic Headache. J Orthop Sport Phys Ther. 2007;37(3):100-7. doi: 10.2519/ jospt.2007.2379.

18. Jull G. Diagnosis of Cervicogenic Headache. J Man Manip Ther. 2006;14(3):136-8. doi: 10.1007/ s10194-006-0277-3.

19. Zito G, Jull G, Story I. Clinical tests of musculoskeletal dysfunction in the diagnosis of cervicogenic headache. Man Ther. 2006;11(2):118-29. doi: 10.1016/j. math.2005.04.007.
20. Hall TM, Briffa K, Hopper D, Robinson K. Comparative analysis and diagnostic accuracy of the cervical flexion-rotation test. J Headache Pain. 2010;11(5):391-7. doi: 10.1007/s10194010-0222-3.

21. Chaves T, Nagamine $H$, Belli J, de Hannai $M$, Bevilaqua-Grossi D, de Oliveira A. Confiabilidade da fleximetria e goniometria na avaliação da amplitude de movimento cervical em crianças. Rev Bras Fisioter. 2008;12(4):283-9. doi: 10.1590/ S1413-35552008000400006.

22. Whitcroft KL, Massouh $L$, Amirfeyz R, Bannister $\mathrm{G}$. Comparison of methods of measuring active cervical range of motion. Spine. 2010;35(19):E976-E980. doi: 10.1097/ BRS.0b013e3181cd6176. 


\section{Anexos}

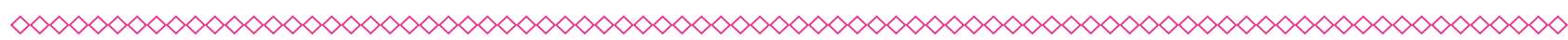

Tabela 1 - Caracterização do perfil da amostra, n=15. São Leopoldo/RS, 2016.

\begin{tabular}{|c|c|c|c|c|c|}
\hline \multirow{3}{*}{ Variáveis } & \multicolumn{4}{|c|}{ Grupo* } & \multirow{3}{*}{$\mathbf{p}$} \\
\hline & \multicolumn{2}{|c|}{ GP $(n=8)$} & \multicolumn{2}{|c|}{ GA $(n=7)$} & \\
\hline & $\mathbf{n}$ & $\%$ & $\mathbf{n}$ & $\%$ & \\
\hline \multicolumn{6}{|l|}{ Sexo } \\
\hline Masculino & 2 & 25,0 & 2 & 28,6 & \multirow[t]{2}{*}{$>0,999 \S$} \\
\hline Feminino & 6 & 75,0 & 5 & 71,4 & \\
\hline Idade (anos) & \multirow{2}{*}{\multicolumn{2}{|c|}{$25,7 \pm 5,1(20-36)$}} & & & \multirow{2}{*}{$0,799 €$} \\
\hline Média $\pm D P$ (Amplitude) & & & & $(21-40)$ & \\
\hline \multicolumn{6}{|l|}{ Trabalha } \\
\hline Sim & 2 & 25,0 & 4 & 57,1 & \multirow[t]{2}{*}{$0,315 \S$} \\
\hline Não & 6 & 75,0 & 3 & 42,9 & \\
\hline \multicolumn{5}{|l|}{ Profissão/Ocupação } & \multirow{3}{*}{$0,315 \S$} \\
\hline Estudante & 6 & 75,0 & 3 & 42,9 & \\
\hline Operário/Estudante & 2 & 25,0 & 4 & 57,1 & \\
\hline
\end{tabular}

*Percentuais obtidos com base no total de cada classificação de cada grupo;

$\S$ : Teste Exato de Fisher;

$€$ : Teste t-Student para grupos independentes assumindo heterogeneidade de variâncias;

Fonte: Elaborada pelos autores. 
Tabela 2 - Critérios de diagnóstico da CTT, n=15. São Leopoldo/RS, 2016.

\begin{tabular}{|c|c|c|c|c|c|}
\hline \multirow{3}{*}{ Variáveis } & \multicolumn{4}{|c|}{ Grupo* } & \multirow{3}{*}{$\mathbf{p}$} \\
\hline & \multicolumn{2}{|c|}{ GP $(n=8)$} & \multicolumn{2}{|c|}{ GA $(n=7)$} & \\
\hline & $\mathbf{n}$ & $\%$ & $\mathbf{n}$ & $\%$ & \\
\hline \multicolumn{6}{|c|}{ Hábito Postural Predominante } \\
\hline Sentada & 6 & 75,0 & 4 & 57,1 & \multirow[t]{2}{*}{$0,608 \S$} \\
\hline Sentada/em pé & 2 & 25,0 & 3 & 42,9 & \\
\hline Número de Crises & \multirow{2}{*}{\multicolumn{2}{|c|}{$31,5 \pm 26,7$}} & & & \multirow{3}{*}{0,0179} \\
\hline Média $\pm D P$ & & & \multicolumn{2}{|c|}{$72,8 \pm 29,8$} & \\
\hline Mediana (Amplitude) & \multicolumn{2}{|c|}{$90,0(10-90)$} & 100 & $30-100)$ & \\
\hline \multicolumn{6}{|l|}{ Duração das Crises } \\
\hline Dias & 3 & 37,5 & 4 & 57,1 & \multirow{3}{*}{$>0,999 \S$} \\
\hline Horas & 4 & 50,0 & 3 & 42,9 & \\
\hline Horas/Dias & 1 & 12,5 & & & \\
\hline \multicolumn{6}{|c|}{ Região Anatômica Acometida ${ }^{A}$} \\
\hline Frontal & 8 & 100,0 & 6 & 85,7 & \multirow{3}{*}{$0,156 \xi$} \\
\hline Occipital & 5 & 62,5 & 4 & 57,1 & \\
\hline Temporal & 3 & 37,5 & 2 & 28,6 & \\
\hline \multicolumn{5}{|l|}{ Caráter Bilateral } & \multirow{2}{*}{---} \\
\hline Sim & 8 & 100,0 & 7 & 100,0 & \\
\hline \multicolumn{6}{|l|}{ Tipo de Dor ${ }^{A}$} \\
\hline Aperto & 3 & 37,5 & 4 & 57,1 & \multirow{3}{*}{$0,103 \xi$} \\
\hline Pressão & 6 & 75,0 & 4 & 57,1 & \\
\hline Peso & 2 & 25,0 & 1 & 14,3 & \\
\hline \multicolumn{6}{|l|}{ Agrava na Atividade Física } \\
\hline Não & 8 & 100,0 & 7 & 100,0 & --- \\
\hline \multicolumn{6}{|l|}{ Náuseas/Vômitos } \\
\hline Não & 8 & 100,0 & 7 & 100,0 & --- \\
\hline \multicolumn{6}{|l|}{ Fotofobia } \\
\hline Sim & 3 & 37,5 & 2 & 28,6 & $>0,999 €$ \\
\hline Não & 5 & 62,5 & 5 & 71,4 & \\
\hline \multicolumn{6}{|l|}{ Recorre a Medicamentos? } \\
\hline Não & & & 2 & 28,6 & $0,200 €$ \\
\hline Sim & 8 & 100,0 & 5 & 71,4 & \\
\hline
\end{tabular}

*Percentuais obtidos com base no total de cada grupo;

A: Percentuais obtidos pela análise do número de ocorrência (múltiplas repostas);

$\S:$ Teste Exato de Fisher; $\mathbf{9}$ : Teste de Mann Whitney; $\xi$ : Teste Q de Cochran; €: Teste Qui-quadrado de Pearson. Fonte: Elaborada pelos autores. 
Tabela 3 - Avaliação e reavaliação da amplitude de movimento nos padrões de movimentos da coluna cervical e cabeça, n=15. São Leopoldo/RS, 2016.

\begin{tabular}{|c|c|c|c|c|c|c|c|}
\hline \multirow{3}{*}{$\begin{array}{c}\text { Variáveis } \\
\text { A - Avaliação } \\
\text { R - Reavaliação }\end{array}$} & \multicolumn{6}{|c|}{ Grupo } & \multirow{3}{*}{$\mathbf{p}$} \\
\hline & \multicolumn{3}{|c|}{ GP $(n=8)$} & \multicolumn{3}{|c|}{ GA $(n=7)$} & \\
\hline & Média & $\begin{array}{l}\text { Desvio } \\
\text { padrão }\end{array}$ & Mediana & Média & $\begin{array}{l}\text { Desvio } \\
\text { padrão }\end{array}$ & Mediana & \\
\hline ADM flexão A & 47,5 & 10,8 & 48,0 & 47,9 & 8,4 & 45,0 & $1,000 €$ \\
\hline ADM flexão R & 50,9 & 11,2 & 52,0 & 53,6 & 10,5 & 52,0 & $0,601 ¥$ \\
\hline $\mathbf{p} z$ & & 0,027 & & & 0,025 & & \\
\hline ADM Extensão A & 44,0 & 5,2 & 42,0 & 46,9 & 4,1 & 50,0 & $0,240 €$ \\
\hline ADM Extensão R & 46,0 & 4,5 & 47,0 & 46,6 & 4,7 & 50,0 & $0,799 ¥$ \\
\hline $\mathbf{p z}$ & & 0,109 & & & 0,655 & & \\
\hline ADM Flx lateral D A & 34,1 & 5,2 & 33,0 & 37,4 & 4,4 & 40,0 & $0,187 €$ \\
\hline ADM Flx lateral D R & 37,8 & 2,7 & 38,0 & 39,1 & 1,6 & 40,0 & $0,228 €$ \\
\hline pz & & 0,043 & & & 0,157 & & \\
\hline ADM Flx lateral E A & 37,3 & 3,5 & 39,0 & 38,6 & 2,5 & 40,0 & $0,395 €$ \\
\hline ADM FIx lateral E R & 39,8 & 1,3 & 40,0 & 39,1 & 1,6 & 40,0 & $0,533 ¥$ \\
\hline $\mathbf{p} z$ & & 0,066 & & & 0,157 & & \\
\hline ADM rotação $D A$ & 36,3 & 5,9 & 35,0 & 39,9 & 3,5 & 38,0 & $0,216 €$ \\
\hline ADM rotação $D R$ & 41,3 & 4,9 & 42,0 & 46,0 & 3,1 & 46,0 & $0,047 €$ \\
\hline pz & & 0,011 & & & 0,027 & & \\
\hline ADM rotação E A & 37,8 & 6,2 & 38,0 & 43,9 & 3,8 & 42,0 & $0,053 €$ \\
\hline ADM rotação E R & 42,4 & 7,5 & 42,0 & 48,6 & 4,8 & 49,0 & $0,092 €$ \\
\hline $\mathbf{p z}$ & & 0,011 & & & 0,036 & & \\
\hline
\end{tabular}

$¥$ : Teste t-Student para grupos independentes assumindo homogeneidade de variâncias;

$€$ : Teste t-Student para grupos independentes assumindo heterogeneidade de variâncias;

z: Teste t-Student para dados pareados.

Fonte: Elaborada pelos autores.

(clique para voltar ao texto) 